\title{
A SIMPLE MODEL OF DYNAMIC CLEAVAGE PRODUCING CONSTANT SPEED CRACK PROPAGATION IN A SAMPLE OF FINITE WIDTH*
}

\author{
COLIN ATKINSON ${ }^{\dagger}$
}

\begin{abstract}
A simple model of dynamic cleavage under longitudinal shear deformation is analyzed as an attempt to understand how ancient lapidaries might have produced smooth cleaved surfaces. Attention is concentrated on the time signature of the load applied to the specimen in order to maintain a constant stress intensity factor (or energy release rate) at the crack tip as it propagates away from the location of the applied load at a constant speed. The analysis is also applicable to situations in which fracture requires a time dependent energy release rate. An example of the cleavage of diamond is considered.
\end{abstract}

Key words. dynamic cleavage, longitudinal shear, fast fracture

AMS subject classifications. $74 \mathrm{~B}, 74 \mathrm{R}, 74 \mathrm{~J}, 35,45$

DOI. $10.1137 / 15 \mathrm{M} 104298 \mathrm{X}$

1. Introduction. In a recent paper, Atkinson et al. (2015), a question related to cleavage of diamond was raised in an attempt to explain the smooth surfaces created by lapidaries possibly as far back as 1645 . In that paper we outlined the results of a simple theory intended to address the problem. Here we explain this theory in more detail and with a simple model (a modification of the flint-knapping model of Fonseca, Eshelby, and Atkinson (1971)) explore the characteristics of the striking load produced by the lapidary. For the specific numerical results given below we use appropriate constants for diamond. If we assume that a crack advances such that the energy flow into the crack tip (the energy release rate) is a material constant and that the crack proceeds at a constant speed we attempt to describe how the load should be applied to be consistent with this. An equivalent description would be that the stress intensity at the crack tip was constant in time. There are other physical models which might suggest that the crack propagates in such a way that this intensity factor or energy release rate would be time dependent. These models could also be treated by the model of this paper. As well as a complete analysis of our model problem we concentrate on the time dependence of the load required to maintain a crack propagating into the specimen (cleaving it into two halves) away from the applied load at a constant speed and in equilibrium with a time independent material property, e.g., a crack tip stress intensity factor or a required energy release into the crack to form new surfaces. Useful long and short time approximations are also given.

It is assumed that a crack is produced that splits (cleaves) the specimen into two by impacting the thin end of the specimen. We analyze this by means of a simple model in which the deformation is assumed to be that of longitudinal shear (see Figure 1). A blow which activates the cleavage launches a stress pulse into the specimen which interacts with the moving crack tip. In this model problem the blow provides a displacement in the $\mathrm{z}$ direction which is antisymmetric about $y=0$. We

${ }^{*}$ Received by the editors October 6, 2015; accepted for publication (in revised form) April 19, 2016; published electronically July 21, 2016.

http://www.siam.org/journals/siap/76-4/M104298.html

†Imperial College, Department of Mathematics, Queen's Gate, South Kensington, London, SW7 2AZ, United Kingdom (c.atkinson@imperial.ac.uk). 


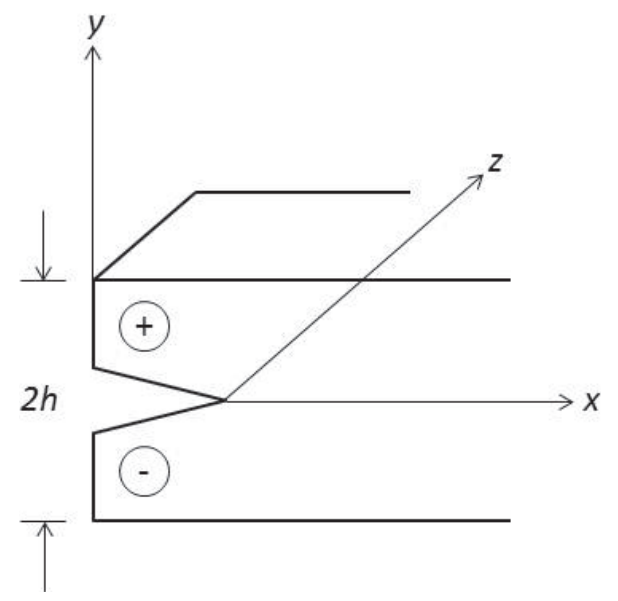

FIG. 1. Schematic of a crack tip traversing a thin specimen of thickness $2 h$ with a stress free boundary on $y= \pm h$.

can thus envisage this as produced by a force $\mp 2 h f^{\prime}(t)$ at $x=x_{1}$ uniformly spread over each half of the specimen edge. The displacement is thus

$$
\begin{aligned}
& u_{30}=-\left(\frac{c_{2}}{\mu}\right) f\left(t+\frac{\left(x-x_{1}\right)}{c_{2}}\right), \quad x<x_{1}, \quad y>0, \\
& =+\left(\frac{c_{2}}{\mu}\right) f\left(t+\frac{\left(x-x_{1}\right)}{c_{2}}\right), \quad x<x_{1}, \quad y<0 .
\end{aligned}
$$

The cleaved plane shears on $y=0$. We suppose the end of the specimen is at $x_{1}=0$. To simplify the analysis we assume the cut, etc., extends to the left so we have the displacement produced

$$
\begin{aligned}
& u_{30}=-\left(\frac{c_{2}}{\mu}\right) f\left(t-\frac{x}{c_{2}}\right) H\left(t-\frac{x}{c_{2}}\right), \quad y>0, \quad x>0, \\
& =-\left(\frac{c_{2}}{\mu}\right) f\left(t+\frac{x}{c_{2}}\right) H\left(t+\frac{x}{c_{2}}\right), \quad y>0, \quad x<0,
\end{aligned}
$$

and the same with opposite sign for $y<0$. The specimen is assumed to have thickness $2 h$. We assume that the crack starts to move with constant speed $v$ when the pulse first hits it at $t=0$. We use the moving coordinate

$$
x^{\prime}=x-v t
$$

and assume antisymmetry about $y=0$.

If the head of the pulse has not yet reached the crack tip then $u_{30}$ is given by (1). If, however, the solution (1) has evolved until the wave has overtaken the crack then the solution (1) will give a discontinuity in the mode three displacement $u_{30}$ ahead of the crack. The correct solution to our problem will be

$$
u_{3}=u_{30}+u_{3}^{\prime},
$$

where $u_{3}^{\prime}$ has to be found to correct for this discontinuity leaving the total displacement and stress continuous ahead of the crack. Also, of course, the crack is stress 
free. Thus we have the equation

$$
\nabla^{2} u_{3}=\frac{1}{c_{2}^{2}} \frac{\partial^{2} u_{3}}{\partial t^{2}}
$$

which becomes in terms of $x^{\prime}$ (defined in (3))

$$
\frac{\partial^{2} u_{3}}{\partial x^{\prime 2}}+\frac{\partial^{2} u_{3}}{\partial y^{2}}-\frac{1}{c_{2}^{2}}\left(\frac{\partial}{\partial t}-v \frac{\partial}{\partial x^{\prime}}\right)^{2} u_{3}=0
$$

(using the antisymmetry about $y=0$ ) with the boundary conditions

$$
\begin{array}{rrrr}
u_{3}^{\prime}+u_{30}=0, \quad x^{\prime}>0 \quad \forall t & y=0, \\
\tau_{y z}=\mu_{3} \frac{\partial u_{3}}{\partial y}=\mu_{3} \frac{\partial u_{3}^{\prime}}{\partial y}=0, \quad x^{\prime}<0 \quad \forall t & y=0, \\
\tau_{y z}=0, \quad y=h & \forall x^{\prime}, t .
\end{array}
$$

In terms of $x^{\prime},(2)$ gives

$$
\begin{array}{ll}
u_{30}=-\left(\frac{c_{2}}{\mu}\right) f\left[\left(1-\frac{v}{c_{2}}\right) t-\frac{x^{\prime}}{c_{2}}\right] H\left[\left(1-\frac{v}{c_{2}}\right) t-\frac{x^{\prime}}{c_{2}}\right], & x^{\prime}>-v t, \\
u_{30}=-\left(\frac{c_{2}}{\mu}\right) f\left[\left(1+\frac{v}{c_{2}}\right) t+\frac{x^{\prime}}{c_{2}}\right] H\left[\left(1+\frac{v}{c_{2}}\right) t+\frac{x^{\prime}}{c_{2}}\right], & x^{\prime}<-v t .
\end{array}
$$

$H(u)$ is the Heaviside function defined as $H(u)=1, u>0$ and $H(u)=0, u<0$.

We extend the definitions in (7) on $y=0$ to include the regions $x^{\prime}<0$ and $x^{\prime}>0$, respectively, and Fourier transform over all $x^{\prime}$ and Laplace transform over $t$ from zero to infinity. Thus

$$
\begin{array}{rr}
\int_{0}^{+\infty} e^{-p t} \mathrm{~d} t \int_{-\infty}^{0}\left(u_{3}^{\prime}+u_{30}\right) e^{i \zeta x^{\prime}} \mathrm{d} x^{\prime}=U_{-}(\zeta, p) & \text { for } \operatorname{Im} \zeta<0 \\
& \equiv \overline{\bar{u}}_{3}^{\prime}+\overline{\bar{u}}_{30},
\end{array}
$$

where the superscript double overbar means Fourier-Laplace transformed. Applying this Fourier-Laplace transform to $u_{30}$ gives from the definition given in (8) the integral over $x^{\prime}$ consisting of two parts, one from $-v t$ to $\left(c_{2}-v\right) t$ and one from $-\left(c_{2}+v\right) t$ to $-v t$ with the appropriate value of the argument of $f$ defined for each part. Changing the variables in the inner $x^{\prime}$ integrals to that of the appropriate argument of the $f$ functions reduces them to

$$
\int_{0}^{t} f(u)\left(e^{i \zeta\left(\left(c_{2}-v\right) t-c_{2} u\right)}+e^{i \zeta\left(\left(-\left(c_{2}+v\right) t+c_{2} u\right)\right.}\right) \mathrm{d} u ;
$$

finally, integrating over $\mathrm{t}$ gives the result below where the multiplying factor $1 / \mu$ has been absorbed into $\bar{f}$ :

$$
\overline{\bar{u}}_{30}=c_{2} \frac{\bar{f}(p+i v \zeta)}{\left(p+i \zeta\left(v+c_{2}\right)\right)}+c_{2} \frac{\bar{f}(p+i v \zeta)}{\left(p+i \zeta\left(v-c_{2}\right)\right)} .
$$

If we add and subtract the value of the pole in the minus region at $\zeta=-\frac{i p}{c_{2}-v}$ we can rewrite

$$
\overline{\bar{u}}_{30}=J_{-}-\frac{\bar{f}\left(\frac{c_{2} p}{c_{2}-v}\right)}{\left(1-\frac{v}{c_{2}}\right) i\left(\zeta+\frac{i p}{c_{2}-v}\right)} .
$$


The first term is a minus function regular in $\operatorname{Im} \zeta<\frac{p}{\left(c_{2}+v\right)}$, the second term being a plus function analytic in $\operatorname{Im} \zeta>-\frac{p}{\left(c_{2}-v\right)}$. This follows because $\bar{f}(p+i v \zeta)$ is analytic in the region $\operatorname{Real}(p+i v \zeta)>0$ as a property of the Laplace transform and hence is a minus function in the complex $\zeta$ plane. From (9)

$$
\text { on } y=0 \quad \overline{\bar{u}}_{3}^{\prime}=U_{-}(\zeta, p)-\overline{\bar{u}}_{30}
$$

and from $(7)_{2}$ we have after Laplace-Fourier transforming the boundary conditions for $\tau_{y z}$ the result

$$
\text { on } y=0 \quad \overline{\bar{\tau}}_{y z}=\int_{0}^{+\infty} e^{-p t} \mathrm{~d} t \int_{0}^{+\infty} e^{i \zeta x^{\prime}} \tau_{y z}\left(x^{\prime}, t\right) \mathrm{d} x^{\prime}=\tau_{+}(\zeta, p)
$$

which will be regular in some upper half-plane $\operatorname{Im} \zeta>-\zeta_{0}$ for some $\zeta_{0}$. If we now Laplace-Fourier transform (6) and use the boundary condition on $y=h$ we write

$$
\overline{\bar{u}}_{3}^{\prime}(\zeta, p)=A \cosh [(h-y) \gamma], \quad 0<y \leq h,
$$

where

$$
\gamma^{2}=\zeta^{2}+\frac{(p+i v \zeta)^{2}}{c_{2}^{2}}
$$

Equation (13) automatically satisfies $(7)_{3}$. From (11) we have

$$
A \cosh (h \gamma)=U_{-}(\zeta, p)-\overline{\bar{u}}_{30}
$$

and from (12)

$$
-\gamma A \sinh (h \gamma)=\frac{1}{\mu} \tau_{+}(\zeta, p)
$$

Eliminating the unknown A between (15) and (16) we obtain

$$
-\frac{1}{\mu} \tau_{+} \frac{\operatorname{coth}(\gamma h)}{\gamma}=U_{-}(\zeta, p)-\overline{\bar{u}}_{30}
$$

holding in some appropriate strip of the complex $\zeta$ plane.

In the equations above we have used the Laplace transform over $t$

$$
\bar{F}=\bar{F}\left(x^{\prime}, y, p\right)=\int_{0}^{+\infty} e^{-p t} F\left(x^{\prime}, y, t\right) \mathrm{d} t
$$

and the Fourier transform over $x^{\prime}$

$$
\overline{\bar{F}}(\zeta, y, \zeta)=\int_{-\infty}^{+\infty} e^{i \zeta x^{\prime}} \bar{F}\left(x^{\prime}, y, p\right) \mathrm{d} x^{\prime} .
$$

In the equations immediately leading up to (17) the transforms are evaluated on $y=0$.

We anticipate that (17) will hold in some strip of the complex $\zeta$ plane defined by the overlap region of the half-planes of regularity of the functions $\tau_{+}$and $U_{-}$. A key 
feature of the solution of the functional equation (17) is to write the functions

$$
F(\zeta)=\frac{\operatorname{coth}(\gamma h)}{\gamma} \equiv F_{+}(\zeta) F_{-}(\zeta)
$$

as the product of two functions regular and nonzero in respective half-planes. If we assume this has been accomplished (see the appendix) then (17) can be rearranged as

$$
-\frac{1}{\mu} \tau_{+} F_{+}=\frac{U_{-}}{F_{-}}-\frac{\overline{\bar{u}}_{30}}{F_{-}},
$$

the last term being, on account of (11), a mixture of plus and minus functions. Fortunately, this can be rearranged as the sum of a plus and a minus function by inspection to give, after rearrangement,

$$
\begin{aligned}
& -\frac{1}{\mu} \tau_{+} F_{+}-\frac{\bar{f}\left(\frac{c_{2} p}{c_{2}-v}\right)}{\left(1-\frac{v}{c_{2}}\right) i\left(\zeta+\frac{i p}{c_{2}-v}\right) F_{-}\left(\frac{-i p}{c_{2}-v}\right)} \\
& \quad=\frac{U_{-}}{F_{-}}-\frac{J_{-}}{F_{-}}+\frac{\overline{c_{2} p}}{\left(1-\frac{v}{c_{2}-v}\right) i\left(\zeta+\frac{i p}{c_{2}-v}\right)}\left[\frac{1}{F_{-}(\zeta)}-\frac{1}{\left.F_{-}\left(\frac{-i p}{c_{2}-v}\right)\right)}\right] .
\end{aligned}
$$

From the factorizsation results of the appendix we have

$$
\lim _{|\zeta| \rightarrow \infty} F_{ \pm}(\gamma)=\left\{\begin{array}{l}
\left(1-\frac{v^{2}}{c_{2}^{2}}\right)^{-\frac{1}{2}} \zeta_{+}^{-\frac{1}{2}} \\
\zeta_{-}^{-\frac{1}{2}}
\end{array}\right.
$$

where the limits are taken in respective + and - regions. Thus from $(22)$ we have

$$
\lim _{|\zeta| \rightarrow \infty} \frac{1}{\mu} \tau_{+}=\frac{i \bar{f}\left(\frac{c_{2} p}{c_{2}-v}\right)\left(1-\frac{v^{2}}{c_{2}^{2}}\right)^{\frac{1}{2}}}{\zeta_{+}^{\frac{1}{2}}\left(1-\frac{v}{c_{2}}\right) F_{-}\left(\frac{-i p}{c_{2}-v}\right)}
$$

and

$$
\lim _{|\zeta| \rightarrow \infty} U_{-}=\frac{\bar{f}\left(\frac{c_{2} p}{c_{2}-v}\right)}{i \zeta_{-}^{\frac{3}{2}}\left(1-\frac{v}{c_{2}}\right) F_{-}\left(\frac{-i p}{c_{2}-v}\right)} .
$$

Using Tauberian theorems for the Fourier transform we can invert the above limiting results giving

$$
\lim _{x^{\prime} \rightarrow 0_{+}} \frac{1}{\mu} \bar{\tau}_{y z}=\frac{1}{\sqrt{\pi}} e^{\frac{i \pi}{4}} \bar{f}\left(\frac{c_{2} p}{c_{2}-v}\right)\left(\frac{1+\frac{v}{c_{2}}}{1-\frac{v}{c_{2}}}\right)^{\frac{1}{2}} \frac{\left(x^{\prime}\right)^{-\frac{1}{2}}}{F_{-}\left(\frac{-i p}{c_{2}-v}\right)}
$$

and

$$
\lim _{x^{\prime} \rightarrow 0_{-}} U_{-}=\frac{\frac{2}{\sqrt{\pi}}\left(-x^{\prime}\right)^{\frac{1}{2}} e^{\frac{i \pi}{4}} \bar{f}\left(\frac{c_{2} p}{c_{2}-v}\right)}{\left(1-\frac{v}{c_{2}}\right) F_{-}\left(\frac{-i p}{c_{2}-v}\right)} .
$$


Note

$$
\frac{e^{\frac{i \pi}{4}}}{F_{-}\left(\frac{-i p}{c_{2}-v}\right)}=p^{\frac{1}{2}}\left(\frac{2 c_{2}}{c_{2}^{2}-v^{2}}\right)^{\frac{1}{2}} \exp \left(-G_{-}\left(\frac{-i p}{c_{2}-v}\right)\right)
$$

where

$$
G_{-}\left(\frac{-i p}{c_{2}-v}\right)=-\frac{1}{2 \pi i} \int_{-\infty}^{+\infty} \frac{\log (\operatorname{coth}(\gamma h))}{\left[\zeta+\frac{i p}{\left(c_{2}-v\right)}\right]} \mathrm{d} \zeta .
$$

We know that on the real $\zeta$ axis with $\zeta=\xi+i \eta,(\xi, \eta)$ real, that in the cut plane

$$
\begin{aligned}
& \text { (32) } \gamma=\left(1-\frac{v^{2}}{c_{2}^{2}}\right)^{\frac{1}{2}}\left(\xi^{2}+\frac{p^{2}}{\left(c_{2}+v\right)^{2}}\right)^{\frac{1}{4}}\left(\xi^{2}+\frac{p^{2}}{\left(c_{2}-v\right)^{2}}\right)^{\frac{1}{4}} \exp \left(i \frac{\left(\theta_{1}+\theta_{2}\right)}{2}\right), \\
& (33) \theta_{1}=-\arctan \left(\frac{p}{\xi\left(c_{2}+v\right)}\right)=-\arctan \left(\xi, \frac{p}{\left(c_{2}+v\right)}\right) \\
& \text { (34) } \theta_{2}=\arctan \left(\frac{p}{\xi\left(c_{2}-v\right)}\right)=+\arctan \left(\xi, \frac{p}{\left(c_{2}-v\right)}\right), \\
& \text { (35) } \gamma=\left(1-\frac{v^{2}}{c_{2}^{2}}\right)^{\frac{1}{2}} p\left(\xi_{1}^{2}+\frac{1}{\left(c_{2}+v\right)^{\frac{1}{2}}}\right)^{\frac{1}{4}}\left(\xi_{1}^{2}+\frac{1}{\left(c_{2}-v\right)^{\frac{1}{2}}}\right)^{\frac{1}{4}} \exp \left(i \frac{\left(\theta_{1}+\theta_{2}\right)}{2}\right) \\
& \text { (36) } \theta_{1}=-\arctan \left(\frac{1}{\left(c_{2}+v\right) \xi_{1}}\right)=\arctan \left(\xi_{1}, \frac{-1}{\left(c_{2}+v\right)}\right) \\
& \text { (37) } \theta_{2}=\arctan \left(\frac{1}{\left(c_{2}-v\right) \xi_{1}}\right)=\arctan \left(\xi_{1}, \frac{-1}{\left(c_{2}-v\right)}\right)
\end{aligned}
$$

where we have written $\xi=p \xi_{1}$.

The specimen is assumed to have thickness $2 h$ and to be much longer in the $z$ direction. We assume that the crack starts to move with constant speed $v$ when the pulse first hits it at $t=0$. If the head of the pulse has not yet reached the crack tip then $u_{30}$ is given by (1). If, however, the solution (1) has evolved while the wave has overtaken the crack then the solution (1) will give a discontinuity in the mode 3 displacement $u_{30}$ ahead of the crack. This is taken into account in the analysis of the problem. The solution to the problem has required writing the displacement as $u_{3}=u_{30}+u_{3}^{\prime}$, where $u_{3}^{\prime}$ has been found to correct for this discontinuity leaving the total displacement and stress continuous ahead of the crack, the crack being, of course, stress free. For our model problem this required solving the wave equation in the displacement $u_{3}$ with shear wave speed $c_{2}$ subject to stress free boundary conditions on the sides of the sample (at $y= \pm h$ ) and a stress free crack running at speed $v$ on $y=0$.

With a coordinate $x^{\prime}=x-v t$ moving with the crack tip, after some analysis we find the Laplace transform of the stress intensity factor at the crack tip is

$$
\bar{K}=\frac{2 c_{2}^{\frac{1}{2}}}{\left(c_{2}-v\right)} \bar{Q}(p) \text {. }
$$

Where we define $\bar{K}$ by

$$
\lim _{x^{\prime} \rightarrow 0_{+}} \frac{1}{\mu} \bar{\tau}_{y z}=\frac{\bar{K}}{\sqrt{ }\left(2 \pi x^{\prime}\right)}
$$


as $x^{\prime}$, the distance from the crack tip, tends to zero. The energy release rate into the crack tip Atkinson and Eshelby (1968) will be

$$
G=\frac{2 c_{2}^{2}}{\left(c_{2}-v\right)^{2}\left(c_{2}^{2}-v^{2}\right)^{\frac{1}{2}}} Q^{2}(t),
$$

where $Q(t)$ is the inverse of $\bar{Q}(p)$ with

$$
\bar{Q}(p)=p^{\frac{1}{2}} \bar{f}\left(\frac{c_{2} p}{c_{2}-v}\right) \exp \left(-G_{-}\left(\frac{-i p}{c_{2}-v}\right)\right)
$$

and

$$
G_{-}\left(\frac{-i p}{c_{2}-v}\right)=\frac{-1}{2 \pi i} \int_{-\infty}^{+\infty} \frac{\log (\operatorname{coth}(\gamma h))}{\zeta+\frac{i p}{\left(c_{2}-v\right)}} \mathrm{d} \zeta
$$

If we use Cauchy's theorem and move the contour with the change of variable

$$
\zeta=\left(c_{2} z-i v\right) p /\left(c_{2}^{2}-v^{2}\right)
$$

then we find

$$
G_{-}\left(\frac{-i p}{c_{2}-v}\right)=\frac{1}{\pi} \int_{0}^{\infty} \frac{\log \left(\operatorname{coth}\left(\frac{h p \sqrt{ }\left(1+z^{2}\right)}{\sqrt{ }\left(c_{2}^{2}-v^{2}\right)}\right)\right)}{1+z^{2}} \mathrm{~d} z
$$

since

$$
\gamma^{2}=\zeta^{2}+\frac{(p+i v \zeta)^{2}}{c_{2}^{2}}
$$

These formulas are derived with the boundary condition that the sides $y= \pm h$ are stress free. To obtain values for $Q(t)$ and hence of the energy release rate we could numerically evaluate the function $G_{-}$and the inverse of $\bar{Q}(p), p$ being the Laplace transform variable. From the above expressions we can show that the energy flow into the moving crack tip is proportional to

$$
\left[\frac{\left(1-v / c_{2}\right)}{\left(1+v / c_{2}\right)}\right] \sigma_{a}^{2} F(t)
$$

where $F(t)$ involves inverting the above integrals. We work with a variable $t_{1}$ defined as

$$
t_{1}=\frac{\sqrt{\left(c_{2}^{2}-v^{2}\right)}}{h} t
$$

and we define $p_{1}$ by

$$
p=\frac{\sqrt{\left(c_{2}^{2}-v^{2}\right)}}{h} p_{1}
$$

hence the result

$$
p t=p_{1} t_{1} .
$$

If in the integral for $G_{-}$we make a change of variable $\zeta=\tan (\theta)$ we can write

$$
G_{-}\left(\frac{-i p}{c_{2}-v}\right)=\frac{1}{\pi} \int_{0}^{\pi / 2} \log \left[\operatorname{coth}\left(p_{1} \sec (\theta)\right)\right] \mathrm{d} \theta .
$$


2. Loading required to produce a constant stress intensity factor. The results of the last section give the singular stress at the crack tip (37) and the associated (time dependent, Laplace transformed) stress intensity factor $\bar{K}$ given in (37). The corresponding energy release rate (the energy flow into the moving crack tip) is given in (39). To proceed further we need a fracture criterion to balance the energy release or stress intensity factor with a material property which is that required to maintain fracture. Our analysis could be applied to any such fracture criterion; however, the simplest is where it is required to produce a constant stress intensity factor $K_{0}$ or energy release rate $G_{0}$ for the time of the cleavage, then

$$
\bar{K}=K_{0} / p .
$$

$K_{0}$ or $G_{0}$ could be velocity dependent but this is not an issue here since we are assuming a constant crack speed and asking for the corresponding time dependent loading to sustain it. Using the time variable $t_{1}=\frac{c_{2}}{h} \sqrt{ }\left(1-\alpha^{2}\right) t$ defined above we find

$$
\bar{f}\left(p_{1}\right)=B \frac{K_{0} \sqrt{ }\left(c_{2}-v\right)}{2 \sqrt{ }\left(p_{1}^{3}\right)} \exp \left(G_{-}\right)
$$

with

$$
B=\left(\frac{h}{c_{2}}\right)^{\frac{3}{2}}\left(\frac{1-\alpha}{1+\alpha}\right)^{\frac{3}{4}},
$$

where the transform is over the time variable $t_{1}$ and $G_{-}$is defined as

$$
G_{-}\left(\frac{-i p}{c_{2}-v}\right)=\frac{1}{\pi} \int_{0}^{\pi / 2} \log \left(\operatorname{coth}\left(p_{1} \sec (\theta)\right)\right) \mathrm{d} \theta .
$$

In all the above equations $v=\alpha c_{2}$. We are interested here in the time signature of the striking blow $f\left(t_{1}\right)$; this is given by the Laplace inverse of $\frac{1}{\sqrt{ }\left(p_{1}^{3}\right)} \exp \left(G_{-}\right)$. To enable an accurate evaluation of $f\left(t_{1}\right)$ for short times we expand both the integral for $G_{-}$in terms of exponentials as well as the expression $\exp \left(G_{-}\right)$in terms of $G_{-}$itself. We invert these expansions term by term taking into account the Heaviside expressions which indicate no effect before a certain time due to various wave reflections from the boundaries of the sample. The inverse Laplace transforms of the powers of $G_{-}$resulting from the expansion of $\exp \left(G_{-}\right)$and the expansion of the integrand $\log \left(\operatorname{coth}\left(p_{1} \sec (\theta)\right)\right)$ of $G_{-}$in terms of exponentials of powers of $\exp \left(-2 p_{1} \sec (\theta)\right)$ again leads to collections of Heaviside functions but now involving sums of secant functions with different dummy $\theta$ arguments and multiple integrals over these arguments. We have taken this up to $\left(G_{-}\right)^{5}$ which is still only accurate for short times and not practical because of the multiple integrals involved. These short time calculations are shown in the figures below which go up to $t_{1}=6$ and $t_{1}=10$. It is interesting to compare with a long time result we obtain by expanding $\frac{1}{\sqrt{ }\left(p_{1}^{3}\right)} \exp \left(G_{-}\right)$for $p_{1}$ small and then inverting to get

$$
f\left(t_{1}\right)=\frac{t_{1}}{\sqrt{ } 2}+\frac{\sqrt{ }(2) \log (2)}{\pi}+O\left(\frac{1}{t_{1}}\right) .
$$

For the specific problem considered here (the lenses discussed in Atkinson et al. $(2015)), t_{c}$ is the time for a constant speed crack of velocity $v$ to traverse the specimen. 
For a specimen of length $30 \mathrm{~mm}$ and half-thickness $1.77 \mathrm{~mm}, t_{c}=\frac{30}{\alpha c_{2}}$ with $v=\alpha c_{2}$ and $c_{2}=11.6 \mathrm{~mm} \mathrm{\mu s}^{-1}$. Thus

$$
t_{1 c}=\frac{30 \sqrt{\left(1-\alpha^{2}\right)}}{1.77 \alpha} .
$$

If one calculates the time at which the first wave leaves the striker and reflects back to the moving crack tip one gets $t=(2 h) / \sqrt{ }\left(c_{2}^{2}-v^{2}\right)$ which translates into $t_{1}=2$ using the $t_{1}$ variable defined above. Note $\alpha=0.99311$ when the crack has just traversed the sample as the reflected wave meets the crack tip and for speeds less than this there are many reflections. The striker would, however, be more likely to do this at the lower crack speeds if he required a smooth surface. It is precisely at these speeds that cracks create atomistically flat mirrorlike surfaces. At higher speeds, rougher, less reflective (mist), and finally very rough surfaces are formed (Buehler and Gao (2006)). It should be noted that our analysis takes into account multiple reflections from the sides of the sample. For example, Figure 2 shows results up to $t_{1}=6$; at $t_{1}=2$ the first reflection from the sides arrives which tends to close the crack so the striker has to increase the time dependence of the load. Prior to the first reflection his loading has to increase proportionally to $\sqrt{ }\left(t_{1}\right)$ in order to maintain a constant applied stress intensity as the crack propagates away from the strike region. Note that at $t_{1 c}=6.15$ the crack has traversed the sample at a speed of $\alpha=.94$; recall that $v=\alpha c_{2}$ is the actual crack speed, $c_{2}$ being the shear wave speed. Note that even at this short time the approximation $f\left(t_{1}\right)=\frac{t_{1}}{\sqrt{ } 2}+\frac{\sqrt{ }(2) \log (2)}{\pi}$ for the time dependence of the striking blow is a good approximation; to see this compare Figure 3. It is interesting to compare this with the asymptotic result derived for long time as $f\left(t_{1}\right)=\frac{t_{1}}{\sqrt{ } 2}+\frac{\sqrt{ }(2) \log (2)}{\pi}$ as in Figure 3. If we consider the time dependence for longer times including more reflections in Figure 4 we plot results up to $t_{1}=10$. For this case $t_{1 c}=10.5$, the crack has traversed the sample at a speed $v=\alpha c_{2}$ with $\alpha=.85$ and the corresponding result for long times is given in Figure 5. For both these examples the switch over from square root to approximately linear time dependence has occurred at approximately $t_{1}=2$ which in the two examples considered is a major fraction of the time to traverse the specimen, being a third and a fifth, respectively, in the two examples. However in the slower crack propagation cases this transient is a very small percentage of the whole loading process, e.g., for $\alpha=.1$ we have $t_{1 c}=168.6$ and so probably is much easier to deal with. Note also, of course, the magnitude of the load required depends on the velocity through the constant $B$ defined above and the critical stress intensity required to fracture the specimen.

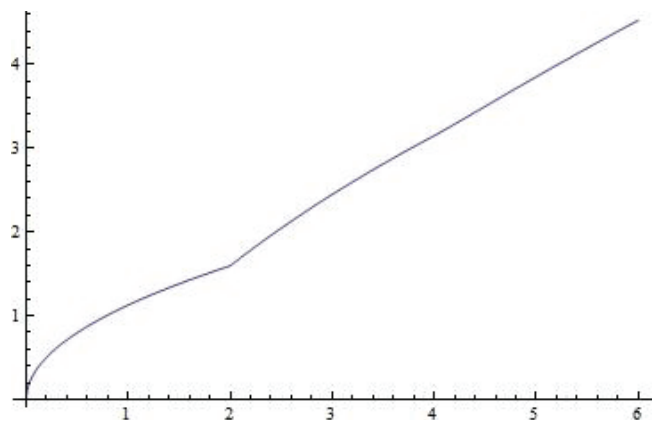

FIG. 2. Applied load versus time $t_{1}$. 


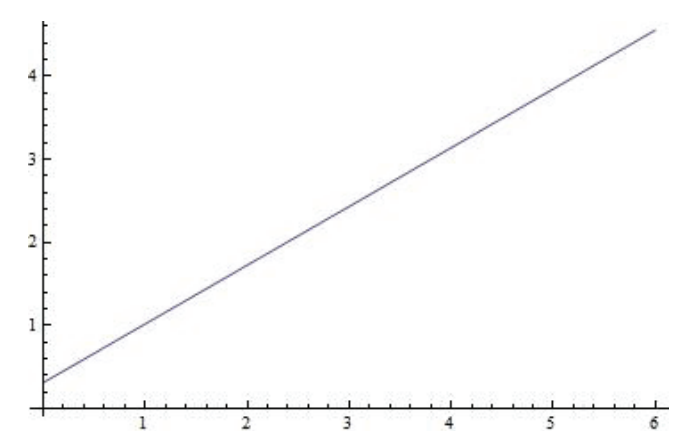

FIG. 3. Applied load versus time $t_{1}$ valid for long times.

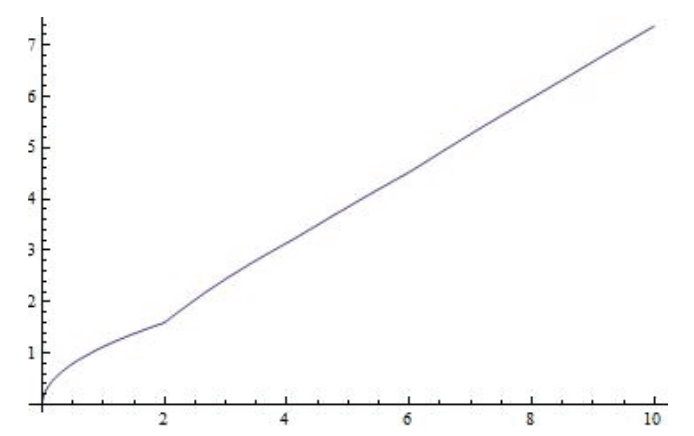

FIG. 4. Applied load versus time $t_{1}$ with more reflections.

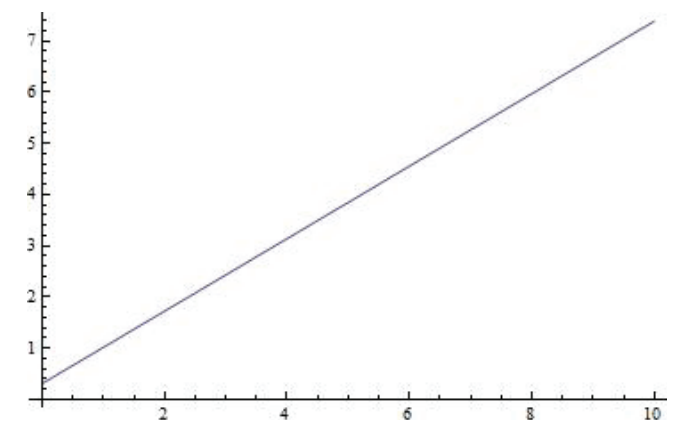

FIG. 5. Applied load versus time $t_{1}$ for long time.

Appendix. From (14) we have

$$
\gamma^{2}(\zeta) \equiv \gamma^{2} \equiv\left(1-\frac{v^{2}}{c_{2}^{2}}\right)\left(\zeta-\frac{i p}{\left(c_{2}+v\right)}\right)\left(\zeta+\frac{i p}{\left(c_{2}-v\right)}\right)
$$

We assume that the crack speed $v$ is less than the wave speed $c_{2}$ and that the Laplace transform variable $p$ is a real positive parameter. Thus we can factorize $\gamma$ as

$$
\gamma=\gamma_{+} \gamma_{-}
$$


where

$$
\begin{aligned}
\gamma_{+} & =\left(1-\frac{v^{2}}{c_{2}^{2}}\right)^{\frac{1}{2}}\left(\zeta+\frac{i p}{\left(c_{2}-v\right)}\right)^{\frac{1}{2}} \\
\text { and } \quad & \gamma_{-}=\left(\zeta-\frac{i p}{\left(c_{2}+v\right)}\right)^{\frac{1}{2}}
\end{aligned}
$$

are analytic in respective upper and lower half-planes cut from $\zeta=\frac{-i p}{\left(c_{2}-v\right)}$ to $-i \infty$ and $\zeta=\frac{+i p}{\left(c_{2}+v\right)}$ to $+i \infty$, respectively.

To factorize $\operatorname{coth}(\gamma h)$ we use Cauchy's theorem and write

$$
\pm \frac{1}{2 \pi i} \int_{-\infty}^{+\infty} \frac{\log (\operatorname{coth}(\gamma h))}{(\zeta-z)} \mathrm{d} \zeta=G_{ \pm}(z)
$$

where the + path lies below the pole at $\zeta=z$ and the - path above and

$$
\log [\operatorname{coth}(\gamma(z) h)]=G_{+}(z)+G_{-}(z) .
$$

Thus

$$
\begin{aligned}
& F_{+}=\gamma_{+}^{-1} \exp \left(G_{+}(\xi)\right) \\
& F_{-}=\gamma_{-}^{-1} \exp \left(G_{-}(\xi)\right)
\end{aligned}
$$

\section{REFERENCES}

J. G. Fonsech, J. D. Eshelby, And C. Atkinson, The fracture mechanics of flint-knapping and allied processes, Int. J. Fracture Mech., 7 (1971), pp. 421-433, doi:10.1007/BF00189112.

C. Atkinson And J. D. Eshelby, The flow of energy into the tip of a moving crack, Int. J. Fracture Mech., 4 (1968), pp. 3-8, doi:10.1007/BF00189137.

C. Atkinson, P. M. Martineau, R. U. A. Khan, J. E. Field, D. Fisher, N. M. Davies, J. V. Samartseva, S. J. Putterman, And J. R. Hird, Cleaving the Halqeh-ye-nur diamonds: A dynamic fracture analysis, Phil. Trans. A, 373 (2015), 20140270, doi:10.1098/rsta.2014.0270.

M. J. Buehler And H. GaO, Dynamical fracture instabilities due to local hyperelasticity at crack tips, Nature, 439 (2006), pp. 307-310, doi:10.1038/nature04408. 